\title{
SINGLY PRODUCED NEW PHYSICS PARTICLES IN FUTURE COLLIDERS
}

\author{
KATRI HUITU \\ Helsinki Institute of Physics, P.O.Box 64, FIN-00014 University of Helsinki, \\ Finland \\ E-mail: katri.huitu@helsinki.fi
}

\begin{abstract}
Single production at hadron and photon colliders of gluinos and sneutrinos of supersymmetric models, as well as radions of the Randall-Sundrum model, is discussed. In the case of supersymmetry, R-parity breaking is needed in order to produce single susy particles. Resonant production of radions is considered at $\gamma \gamma$ collider.
\end{abstract}

The main motivation for considering single production of previously unobserved particles is the lower production threshold when compared to the pair production. Also, there may be enhancement in the cross section, if the new particle can be produced as an $s$-channel resonance.

The most interesting scenarios for new physics from several previous years are supersymmetry and models inspired by the extra dimensions. We will consider here R-parity breaking supersymmetric model and Randall-Sundrum model of extra dimensions 1 . The talk is based on the references ㅂ.

\section{R-parity violation}

The R-parity, $R_{p}=(-1)^{3 B+L+2 S}$ ( $L$ is lepton number, $B$ is baryon number, and $S$ is spin), is often used in the minimal supersymmetric standard model (MSSM) to disallow terms, which would lead to fast proton decay. It is well-known that it would be enough to have either lepton or baryon number conservation instead of the whole R-parity. Adding a subset of the possible R-parity violating $\left(\not R_{p}\right)$ terms,

$$
W_{\not h_{p}}=\lambda_{[i j] k} L_{i} L_{j} \bar{E}_{k}+\lambda_{i j k}^{\prime} L_{i} Q_{j} \bar{D}_{k}+\lambda_{i[j k]}^{\prime \prime} \bar{U}_{i} \bar{D}_{j} \bar{D}_{k}+\epsilon_{i} L_{i} H_{u}
$$

makes it possible to produce superparticles singly, and the lightest supersymmetric particle (LSP) will decay. The violation of R-parity has several interesting phenomenological consequences, which are not allowed in the MSSM, e.g. neutrinos become massive and they mix.

The R-parity violating couplings are bounded by experimental limits. A list of the bounds is reported e.g. in 0 . 


\subsection{The process $p p(p \bar{p}) \rightarrow(t+\bar{t}) \tilde{g}$}

We consider two different schemes. In the first one we assume universal boundary conditions at the GUT scale and radiative symmetry breaking. At oneloop we then have the relation $M_{1} / g_{1}^{2}=M_{2} / g_{2}^{2}=M_{3} / g_{3}^{2}$, which tells us that the gluinos are the heaviest gauginos at the weak scale. The other scenario, which is considered, has a heavy gluino as LSP 6 . There have been worries that this kind of gluino would produce too many energetic neutrinos when annihilating in the sun, but this problem is solved, if R-parity is broken.

In the process also top quarks are produced singly. When compared to the other R-parity breaking single top production processes, which have been studied, the advantage of our calculation is that we have $\lambda^{\prime \prime 2}$, not $\lambda^{\prime \prime 4}$.

Detection possibilities are determined by the decay modes of the produced particles. For top, only the decay $t \rightarrow b W \rightarrow b l \nu$ is considered, since it is experimentally the easiest. The gluino decay patterns are more complicated, see e.g. 6 , and depend on the gluino and other supersymmetric particle masses. If gluino is the LSP or $\lambda^{\prime \prime}$ coupling is large, the decay mode $\tilde{g} \rightarrow q_{i} q_{j} q_{k}$ becomes important. There are two $b$ or $t$ quarks in $60-70 \%$ of the decays, and the final state is $t \tilde{g} \rightarrow 3 b+n l+$ missing energy $(n=1,2,3)$.

Concerning the possible light gluino window around $25 \mathrm{GeV} \lesssim m_{\tilde{g}} \lesssim 35$ $\mathrm{GeV}$, it seems that reanalysing Tevatron Run I data would either close the window or improve the limits on couplings and masses. For heavier gluino, either LSP or not, LHC can put strict constraints on the coupling constants or masses.

\subsection{The process $\gamma \gamma \rightarrow \tilde{\nu}$}

The advantage of the linear collider is its clean background when compared to the hadron colliders. In the $\gamma \gamma$ collision mode, a resonance can be probed over a wide mass range, and couplings involving heavy flavours can be introduced. In addition to being loosely bounded, with an assumption of family symmetry, one may expect the couplings involving heavy flavours to be larger than those involving light flavours.

The productions of $\tilde{\nu}_{2}$ ( $b$ quark or $\tau$ lepton in the loop) and $\tilde{\nu}_{3}$ (b quark in the loop) were studied. If sneutrino and Higgs are of similar mass, the Rparity violating flavour nondiagonal decays are essential for detection. (Also more involved calculation for $\gamma \gamma \rightarrow \chi^{ \pm} l^{\mp}$ was performed, but it is dominated by the resonant sneutrino production.)

Single sneutrino production $\gamma \gamma \rightarrow \tilde{\nu}+f \bar{f}^{\prime}$ with comparable cross section was studied in 9 . The major advantage of this process is that the R-parity violating sources may be distuinguished from the final state.

susy01: submitted to World Scientific on November 10, 2018 


\section{Radions in the process $\gamma \gamma \rightarrow \Phi$}

A recently popular solution to the hierarchy problem is provided by extra dimensions 1 . Stabilization of the modulus leads to a physical light particle, radion 10 . The radion, $\Phi$, may be lighter than the lowest lying Kaluza-Klein modes of graviton, and are thus the first particles, which are typical for the model and possibly accessible in future collider experiments.

In general, radion can mix with Higgs with a mixing parameter $\xi$ 11. The peculiarity of the kinematical mixing is that the resulting mixing matrix is nonunitary, and the matrix elements have discontinuities. Detection of lighter than $8 \rho 0 \mathrm{GeV}$ radion seems possible for $\sqrt{s}=1 \mathrm{TeV}$, if the radion VEV is $1 \mathrm{TeV}$. If mixing is large, also a light radion with $\mathrm{VEV}$ of $10 \mathrm{TeV}$ may be possible to detect. The different decay patterns of radion and Higgs make it possible to distuinghuish between these two particles.

\section{Acknowledgments}

The author thanks the organizers of SUSY'01 in Dubna for an inspiring and well organized meeting. She is grateful to the Academy of Finland (project numbers 163394 and 48787) for financial support.

\section{References}

1. L. Randall, R. Sundrum, Phys. Rev. Lett. 83, 3370 (1999); Phys. Rev. Lett. 83, 4690 (1999).

2. M. Chaichian, K. Huitu, Z.H. Yu, Phys. Lett. B 490, 87 (2000).

3. M. Chaichian, K. Huitu, Z.H. Yu, Phys. Lett. B 508, 317 (2001).

4. M. Chaichian, K. Huitu, A.B. Kobakhidze, Z.H. Yu, hep-ph/0106077, to appear in Phys. Lett. B.

5. B.C. Allanach, A. Dedes, H.K. Dreiner, Phys. Rev. D 60, 075014 (1999).

6. S. Raby, Phys. Rev. D 56, 2852 (1997), Phys. Lett. B4221581998; S. Raby, K. Tobe, Nucl. Phys. B 539, 3 (1999); A. Mafi, S. Raby, Phys. Rev. D 62, 035003 (2000).

7. A. Faroggi, K. Olive, M. Pospelov, Astropart. Phys. 13, 31 (2000).

8. A. Bartl et al, Nucl. Phys. B 502, 19 (1997)

9. M. Chaichian, K. Huitu, S. Roy, Z.H. Yu, hep-ph/0107111.

10. W.D. Goldberger, M.B. Wise, Phys. Rev. Lett. 83, 4922 (1999);Phys. Rev. D 60, 107505 (1999); M. Luty, R. Sundrum, Phys. Rev. D 62, 035008 (2000).

11. G. Giudice, R. Rattazzi, J.D. Wells, Nucl. Phys. B 595, 250 (2001). 